\title{
Competência social de psicóticos: o comportamento de olhar nas fases de escuta e de elocução de interações sociais
}

\author{
Marina Bandeira \\ Elaine Leandro Machado \\ Sabrina Martins Barroso \\ Tais Ribeiro Gaspar \\ Magali Milene Silva \\ Universidade Federal de São João Del Rei
}

\begin{abstract}
Resumo
Investigou-se o comportamento de olhar nas fases de escuta e elocução de interações sociais de 35 pacientes psicóticos comparativamente a 35 sujeitos não-clínicos. Dois observadores treinados observaram os sujeitos em 4 situações sociais de fazer e receber críticas, em desempenho de papéis, gravados em vídeo. Variou-se o tipo de situação e o gênero do interlocutor. Os resultados mostraram maior duração e freqüência de olhar o interlocutor e maior fixidez do olhar no grupo dos pacientes. Ambos os grupos olharam mais o interlocutor na escuta do que na elocução, tal como esperado, tendo os pacientes discriminado menos as fases. Ambos os grupos olharam menos para o interlocutor aos receber do que ao fazer críticas e olharam menos para o interlocutor masculino do que feminino. Estes resultados replicam dados de outros pesquisadores e sugerem uma dificuldade dos pacientes em processos cognitivos envolvidos nas interações sociais e resolução de problemas.
\end{abstract}

Palavras-chave: competência social; psicóticos; contato visual; reinserção social; desempenho de papéis

\begin{abstract}
Social skills of psychotics: gaze during the listening and talking phases of social interactions. This research investigated the characteristics of gaze in the listening and talking phases of social interactions, for 35 psychotics compared to 35 non-clinical subjects. Two judges observed them interacting with trained partners in 4 videotaped role-play situations, regarding giving and receiving criticism. Types of situations and gender of partners were varied. Results showed significant greater duration and frequencies of gaze and more fixed gaze in patients group. Both groups looked more to the partner during listening than talking phase of the interactions, as expected, the patients discriminating less between phases. Both groups looked less in the situations were they have been criticized than in situations where they criticized the partners. Both groups looked less when interacting with male partner, specially the patients. These results in general replicated data from other researchers and indicated which specific dimensions of looking need to be addressed in social skills training for psychiatric patients.

Key words: social skills; gaze, psychotics; desinstitutionalization; role-play
\end{abstract}

$\mathrm{D}$ entre os problemas encontrados no processo de reinserção social dos doentes mentais na comunidade, destaca-se em particular sua dificuldade de funcionamento social, caracterizada pelo isolamento social e distúrbios no desempenho de papéis sociais, assim como dificuldade de estabelecer e manter relações sociais (Foy, Wallace, \& Liberman, 1983; Liberman, 1984). O déficit ou deterioração do funcionamento social tem sido indicado como uma característica dos pacientes psicóticos, em geral, e dos esquizofrênicos em particular, sendo um indicador do prognóstico psiquiátrico. O funcionamento social dos psicóticos após a alta está relacionado ao seu nível de funcionamento social pré-mórbido e interepisódicos (Bellack, Morrison, Mueser, \& Wade, 1989; Mueser, Bellack, Morrison, \& Wixted, 1990a; Mueser, Bellack, Morrison, \& Wade, 1990b; Mueser, Wallace, \& Lieberman, 1995). 
Os pacientes psiquiátricos encontram dificuldades particularmente em enfrentar as situações sociais estressantes (Cyr, Hodgins, Gaston, \& Viens, 1990), o que pode desencadear uma exacerbação de sintomas, que conduz à ocorrência de reinternações. A alta taxa de reinternações constitui um dos problemas mais citados no processo de desinstitucionalização psiquiátrica, conhecido como o Fenômeno da Porta Giratória (Bandeira, 1991; Bandeira \& Dorvil, 1996; Dorvil, 1987). Porém, o número e a gravidade das rehospitalizações dos doentes mentais desinstitucionalizados diminui quando o seu nível de competência social é maior (Donahoe \& Drisienga, 1988; Hogarty et al.,1986; Liberman et al., 1985; Shepperd, 1986). Portanto, o estudo sistemático das dificuldades de habilidades sociais dos pacientes psiquiátricos é necessário para favorecer uma melhor reinserção social.

Pesquisas nesta área mostraram que os pacientes psiquiátricos apresentavam baixa competência social, em estudos transversais e longitudinais (Mueser, Bellack, Douglas, \& Morrison, 1991). Observou-se ainda que os esquizofrênicos apresentavam menor grau de competência social do que os pacientes com desordens afetivas, enquanto estes últimos, por sua vez, possuíam menor grau de competência social do que um grupo de referência não-clínico (Bellack et al., 1989; Bellack, Morrison, Mueser, Wade, \& Sayer, 1990; Mueser et al., 1990b). As diferenças entre os grupos ocorreram em diferentes tipos de situações sociais e envolveram tanto o aspecto verbal da competência social, quanto os componentes não-verbais e paralinguísticos (Mueser et al., 1990a; 1990b; Tremblay, 1992).

O estudo das habilidades sociais de pacientes psiquiátricos tem sido realizado principalmente através da observação sistemática do comportamento dos sujeitos em interações sociais, através de desempenho de papéis (Bellack, 1983; Bellack et al., 1990; Mueser et al., 1990b). As medidas de desempenho de papéis apresentaram maior validade para esta população-alvo, em termos de validade preditiva, discriminante e concomitante, assim como fidedignidade inter e intra-avaliadores (Bellack et al., 1990; Eisler, Hersen, Miller, \& Blanchard, 1975; Merluzzi \& Biever, 1987; Mueser et al., 1990b; Mueser et al., 1991). As situações sociais empregadas são variadas, devido à especificidade situacional das habilidades sociais, que se refere a desempenhos e não a traço (Caballo, 1996; Del Prette \& Del Prette, 1999; Gambrill, 1978). Cyr et al. (1990) destacaram a importância do estudo do comportamento dos pacientes em situações sociais conflituosas, devido às suas dificuldades particulares nestas situações. Tremblay (1992) destacou, em particular, as situações de fazer e receber crítica, que diferenciaram mais o grupo de pacientes de um grupo não-clínico, e que serão utilizadas no presente trabalho.

Pesquisas realizadas em nosso meio também observaram déficits dos pacientes psicóticos nas habilidades sociais verbais, não-verbais e paralinguísticas. Os pacientes apresentaram dificuldades de expressar insatisfação ou necessidade e de solicitar mudanças no comportamento do interlocutor. Apresentaram ainda déficits nos aspectos nãoverbais e paralinguísticos, tais como volume de voz mais baixo, menor duração da fala, maior freqüência e duração de silêncios (Bandeira, Machado, \& Pereira, 2002), freqüência menor de gestos de apoio à fala e afeto menos modulado e menos congruente com a situação (Bandeira, Cardoso, Fernandes, Resende, \& Santos, 1998). Além disso, a clareza, articulação e ritmo da fala estavam igualmente deficitários (Bandeira et al., 1998), assim como a impressão geral de competência social (Bandeira \& Tremblay, 1998) e de auto-afirmação na defesa de seus direitos (Bandeira et al., 2002; Bandeira \& Ireno, no prelo). Portanto, as dificuldades específicas de habilidades sociais de pacientes psiquiátricos transcendem os contextos culturais.

Entretanto, estes resultados obtidos em nosso meio não abordaram toda a amplitude das dificuldades de habilidades sociais dos pacientes psiquiátricos estudadas na literatura desta área. Trower (1980) observa, por exemplo, que os pacientes psiquiátricos apresentam déficits também nas habilidades sociais processuais. Estas habilidades se referem à capacidade do indivíduo de gerar comportamento flexível, em resposta a mudanças nos estímulos sociais dos interlocutores e das situações, requerendo para isto o monitoramento vigilante dos estímulos verbais e não-verbais dos interlocutores, tais como suas expressões e pistas de seu comportamento, utilizando-as para monitorar seu próprio comportamento (Trower, 1980). Indivíduos que apresentam estas habilidades variam mais o seu comportamento de acordo com mudanças ocorridas nas situações, no comportamento dos interlocutores e em função das fases de escuta e de elocução das interações sociais (Argyle \& Kendon, 1967). Os pacientes psiquiátricos apresentam menor variabilidade ou flexibilidade do comportamento frente a mudanças nas situações sociais (Bellack et al., 1990; Moos, 1968). As habilidades processuais envolvem, portanto, o comportamento de olhar, pois este tem uma função importante na detecção e monitoramento dos estímulos sociais presentes em uma interação social (Argyle \& Dean, 1965). Devido às funções diferenciadas do comportamento de olhar em cada fase da interação social, ele deve ser estudado separadamente nas fases de escuta e de elocução (Bellack, 1983, Trower, 1980).

Uma das habilidades sociais processuais focalizadas aqui envolve as relações entre o comportamento de olhar nas fases de escuta e de elocução de uma interação social. Segundo Argyle e Kendon (1967), Argyle e Dean (1965) e Caballo (1996), as pessoas apresentam habitualmente uma inversão entre os comportamentos de olhar e de falar, nas fases de escuta e de elocução. Em uma interação social, o indivíduo olha o seu interlocutor mais tempo durante a fase de escuta (75\% do tempo) do que de elocução (entre $30 \%$ a $50 \%$ do tempo). Além disso, os olhares são mais prolongados na fase de escuta. Esta variação entre as fases indica que o indivíduo é capaz de processar a diferença existente entre os estímulos presentes em uma e outra fase da interação social e que ele é capaz de se ajustar a estas variações, requerendo para isto a percepção e a interpretação dos estímulos interpessoais, o monitoramento dos estímulos sociais e o automonitoramento ( Mueser et al., 1996). Kendon (1967) e Argyle e Dean (1965) 
sugerem igualmente que a diminuição do olhar quando a pessoa está formulando seu pensamento, na fase de elocução, teria a função adaptativa de reduzir a quantidade de informação externa concomitante, favorecendo o processo de elaboração da resposta.

Trower (1980) encontrou um menor grau de inversão entre os comportamentos de olhar e de falar para sujeitos socialmente incompetentes. Ou seja, eles apresentavam uma diferença menor entre as fases de escuta e de elocução do que sujeitos socialmente competentes, no que se refere à proporção da duração do olhar. Segundo Trower (1980), estes resultados indicam um menor grau de habilidades processuais de monitoramento, detecção e interpretação de estímulos sociais. Este déficit pode estar relacionado a dificuldades perceptuais e cognitivas, pois já foi observado que os pacientes com déficits cognitivos possuem menor grau de habilidades sociais (Penny, Mueser, \& North, 1995).

Além das dificuldades citadas acima, o comportamento de olhar de pacientes psicóticos, em particular o esquizofrênico, tem sido descrito como peculiar. Uma maior freqüência de desvios de olhar (averted gaze) tem sido associada à esquizofrenia. Os desvios de olhar têm sido associados igualmente a algumas características de personalidade, tais como introversão, inibição emocional, tensão interna e apreensão e baixa auto-estima (Larsen \& Shackelford, 1996). Segundo Rutter (1976) e Argyle e Kendon (1967), estudos de caso clínico, não controlados, com entrevistas feitas por psiquiatras, indicaram que pacientes esquizofrênicos evitavam o olhar do interlocutor. Por outro lado, em um estudo controlado, envolvendo outro tipo de interlocutor e temas da vida cotidiana, Rutter (1976) obteve resultados contrários, os pacientes esquizofrênicos apresentando maior duração do olhar do que o grupo nãoclínico e maior também do que os grupos de pacientes com diagnósticos de depressão, neurose e/ou distúrbios de personalidade.

Duas variáveis foram levantadas para explicar estes resultados contraditórios: o status do interlocutor e o tema pessoal das interações sociais. Argyle e Kendon (1967) relataram pesquisas demonstrando que assuntos pessoais embaraçosos, processos emocionais e materiais cognitivamente mais difíceis provocavam evitação do olhar. Além disso, os esquizofrênicos evitavam o olhar do interlocutor quando este os encarava ininterruptamente. Segundo Argyle e Dean (1965), olhares prolongados demais, acima da faixa usualmente encontrada (entre 1 e 7 segundos), geram ansiedade nas pessoas. Estes fatores, assim como o status do psiquiatra, poderiam explicar a evitação do olhar de pacientes esquizofrênicos, encontrada em estudos de caso com entrevistas psiquiátricas.

Beaulieu (1996) observou uma outra característica do comportamento de olhar de pacientes psicóticos, que se refere à fixidez do olhar. A autora identificou três diferentes categorias de desvios de olhar, envolvendo três durações distintas. Beaulieu observou que os pacientes apresentavam menos desvios de olhar da categoria de mais curta duração, do que um grupo de referência não-clínico. Estes resultados indicaram, segundo aquela autora, que o olhar dos pacientes era mais fixo, o que poderia afetar suas interações sociais negativamente. Ela concluiu que os seus resultados concordavam com os de Rutter (1976), pois os pacientes olhavam mais para o interlocutor, contrariando os resultados dos estudos de caso não-controlados com entrevistas psiquiátricas.

Em nosso meio, não foram encontradas pesquisas com pacientes psicóticos, visando investigar as características do seu comportamento de olhar. Esta informação, no entanto, é importante para compreender a natureza das dificuldades específicas dos pacientes psiquiátricos nas interações sociais. Dificuldades identificadas nesta área podem fornecer informações da presença de déficits perceptuais e cognitivos dos pacientes. Além disso, o comportamento de olhar é essencial para a comunicação, tendo um impacto importante nas interações sociais. A evitação do olhar ou o olhar com duração excessiva ou fixo demais podem interferir no desempenho de um indivíduo, com repercussões no seu funcionamento social e nas reações das pessoas ao seu comportamento (Larsen \& Shackelford, 1996). O objetivo deste trabalho é investigar as características do comportamento de olhar de pacientes psicóticos, comparativamente a um grupo não-clínico, através da observação sistemática do comportamento, em situações de fazer e receber críticas.

\section{Método}

\section{Participantes}

Participaram desta pesquisa 70 sujeitos do sexo masculino, de nível sócio-econômico baixo, divididos em dois grupos. O grupo clínico compunha-se de 35 pacientes psiquiátricos desinstitucionalizados, com idade média de 39,4 anos, selecionados a partir dos arquivos do hospital psiquiátrico da FHEMIG, em Barbacena, e do Hospital Paulo Menicucci, em Lavras, diagnosticados pelos psiquiatras dos hospitais como CID-295 e CID-298. Todos os sujeitos estavam sendo medicados com drogas antipsicóticas no período da pesquisa. O grupo não-clínico era composto de 35 sujeitos, com idade média de 37,4 anos, sem história psiquiátrica, morando na mesma região da cidade que os pacientes.

\section{Instrumento de medida}

Situações sociais. O comportamento dos sujeitos foi avaliado através do desempenho de papéis em 4 situações, que representavam interações sociais cotidianas entre membros de uma família, gravadas em vídeo. Estas situações fazem parte da Escala de Avaliação de Competência Social, EACS (Bandeira \& Tremblay, 1998) e foram adaptadas e validadas a partir de uma escala canadense de competência social (Tremblay \& Bandeira, 1989). Duas situações eram de fazer crítica e duas de receber crítica. Para cada tipo de situação, variou-se o gênero do interlocutor. Nas situações de fazer crítica, o sujeito deveria expressar insatisfação com o 
comportamento do interlocutor e solicitar mudança do seu comportamento, enquanto que nas situações de receber crítica, ele deveria aceitar a responsabilidade do seu próprio comportamento, mas expressar sua necessidade e propor uma solução que satisfizesse esta necessidade, sem desrespeitar a do outro.

Os sujeitos interagiam com interlocutores previamente treinados, que utilizavam réplicas múltiplas padronizadas, porém flexíveis, que favoreciam uma maior naturalidade nas interações sociais (Bandeira \& Tremblay, 1998; Bandeira et al., 1998; Bellack, 1983).

Categorias de comportamento observadas. A observação sistemática do comportamento foi feita por dois pares de juizes independentes, cada par observando diferentes categorias de comportamentos, separadamente nas fases de escuta e de elocução. A fase de escuta foi definida como os intervalos de tempo em que o sujeito escutava o interlocutor durante sua fala. A fase de elocução foi definida como os intervalos de tempo em que o sujeito falava com o interlocutor. Foram utilizadas as seguintes medidas: 1. Proporção da duração do contato visual por fase: Na fase de escuta: soma dos intervalos de tempo em que o sujeito olhava para o interlocutor, enquanto escutava, dividida pelo tempo de fala do interlocutor. Na fase de elocução: soma dos intervalos de tempo em que o sujeito olhava para o interlocutor, enquanto falava, dividida pelo tempo de fala do sujeito. 2. Freqüência/ minuto do contato visual por fase: Na fase de escuta: soma do número de vezes em que o sujeito olhava para o interlocutor, enquanto o escutava, dividida pelo tempo de fala do interlocutor. Na fase de elocução: soma do número de vezes em que o sujeito olhava para o interlocutor, enquanto falava, dividida pelo tempo de fala do sujeito. 3. Freqüência/ minuto dos desvios de olhar por fase: Na fase de escuta: soma do número de vezes em que o sujeito desviava o olhar do interlocutor enquanto o escutava, dividida pelo tempo de fala do interlocutor. Na fase de elocução: soma do número de vezes em que o sujeito desviava o olhar do interlocutor, enquanto falava, dividida pelo tempo de fala do sujeito. 4 . Freqüencia/ minuto do piscar por fase: Na fase de escuta: número de vezes em que o sujeito fechava e abria os olhos rapidamente, enquanto escutava, dividido pelo tempo de fala do interlocutor. Na fase de elocução: número de vezes em que o sujeito fechava e abria os olhos rapidamente, enquanto falava, dividido pelo tempo de fala do sujeito.

Para efetuar estes cálculos, foram medidas a duração da fala do sujeito e da fala do interlocutor, definidos como a soma dos intervalos de tempo em que o sujeito ou o interlocutor falava, excetuando falas iniciais de cumprimentos e falas finais de despedidas.

\section{Procedimento}

Treinamento dos juízes. Cada par de observadores foi treinado pelo pesquisador, utilizando gravações de sujeitos que não participavam da pesquisa, totalizando 108 interações sociais. As sessões de treinamento eram diárias, durando aproximadamente quatro horas. Os juizes observavam isoladamente os comportamentos de um sujeito e, em seguida, se reuniam com o pesquisador para visionar de novo as cenas, identificando as causas dos desacordos e estabelecendo critérios comuns de avaliação. O treinamento durou 3 meses e foi encerrado quando os pares de juízes atingiram 85\% de acordo entre eles.

Coleta de dados. A coleta de dados era feita diariamente em dois períodos do dia, manhã e tarde, cada período para um par de juizes. Em cada sessão de observação, o juiz 1 observava as situações 1 e 2 de um dado sujeito, em seguida o juiz 2 observava estas mesmas situações. Este procedimento era utilizado, em seguida, para a observação das situações 3 e 4. Observava-se apenas uma categoria de cada vez e um sujeito por dia, para evitar o cansaço dos observadores e favorecer sua concentração e a precisão das medidas. Cada juiz observava sozinho, a fim de evitar a interferência de um juiz sobre o outro. Os juizes estavam “cegos” quanto ao grupo a que pertenciam os sujeitos.

Para aumentar a precisão, as medidas mais difíceis de serem observadas, as de duração e a do comportamento de piscar, eram cronometradas três vezes, sendo que apenas a mediana das três era utilizada como escore. O comportamento de piscar só foi medido nas situações 1 e 3 , de receber críticas, devido à dificuldade de observar este comportamento nas outras situações. Por isto, não se analisou este comportamento em função do tipo de situação, mas apenas em função do gênero do interlocutor.

Após a observação, os juizes se reuniam para calcular a porcentagem de acordos e desacordos obtidos e chegarem a um consenso. Para as medidas de duração, considerava-se que os dois juízes estavam em acordo, quando a diferença entre as suas medidas estava dentro da faixa de 15\%. Caso esta diferença fosse igual ou superior a 15\%, considerava-se como desacordo. Este mesmo critério foi adotado para a categoria freqüência do piscar.

A análise dos dados foi realizada através do programa estatístico SPSS-PC. Foram feitas comparações entre os dois grupos de sujeitos, através da análise de variância (ANOVA). As comparações entre as fases de escuta e de elocução foram feitas através do teste $t$ para amostras emparelhadas. Esta última análise foi feita também para comparar os dados referentes aos dois tipos de situações sociais e ao gênero dos interlocutores.

Fidedignidade das medidas. Para avaliar a fidedignidade das medidas, foram utilizados: 1 . O cálculo da porcentagem de acordo interjuizes, que era feito diariamente para todos os sujeitos. 2. A análise correlacional de Spearman entre os escores de todos os sujeitos, obtidos por cada par de observadores antes das reuniões de consenso.

A porcentagem média de acordo interjuizes foi de 95,67\% para o primeiro par de juizes e de $91,88 \%$ para o segundo par de juizes. A correlação global interjuizes, incluindo todas as categorias de comportamento observadas foi de $r=0,98$ ( $p=$ 
$0,00)$ para o primeiro par de juízes e de $r=0,99(p=0,00)$ para o segundo par de juízes. As correlações interjuizes para cada categoria de comportamento variaram de $r=0,88$ a $r=0,99$ e foram: freqüência do piscar: $r=0,99(p=0,00)$; duração do contato visual: $r=0,99(p=0,00)$; freqüência do contato visual: $r=0,99(p=0,00)$; freqüência do desvio de olhar: $r=0,99(p=$ 0,00); duração da fala do interlocutor: $r=0,88(p=0,00)$; duração da fala do sujeito: $r=0,99(p=0,00)$.

Esta alta fidedignidade das medidas se deve, provavelmente, ao treino prolongado dos juizes, aos critérios específicos e bem definidos de observação e ao fato de se observar apenas um comportamento de cada vez e apenas um sujeito por dia, de modo a favorecer a concentração dos juízes e minimizar o cansaço. O cálculo diário de acordo entre os juízes servia como feedback e incentivo para o desempenho na observação dos sujeitos.

\section{Resultados}

Os dois grupos eram equivalentes com relação à idade, estado civil, renda familiar e per capita, tipo de residência, escolaridade e número de repetições escolares, conferindo maior validade interna à pesquisa (Bandeira \& Tremblay, 1998).

Comparação entre os grupos clínico e não-clínico

As médias e desvios-padrão dos parâmetros do comportamento de cada grupo estão apresentados na Tabela 1, para as fases de escuta e de elocução e no total das interações.

Tabela 1

Médias e desvios-padrão dos parâmetros do comportamento de olhar dos grupos clínico e não-clínico, nas fases de escuta e de elocução e no total das interações sociais

\begin{tabular}{|c|c|c|c|c|c|}
\hline & \multicolumn{4}{|c|}{ Parâmetros do comportamento de olhar } & \multirow[b]{3}{*}{$p$ (grupo) } \\
\hline & \multicolumn{2}{|c|}{ Grupo clínico } & \multirow{2}{*}{$\begin{array}{l}\text { Grupo não } \\
\text { Média }\end{array}$} & \multirow{2}{*}{$\begin{array}{l}\text {-clínico } \\
\text { Desvio - } \\
\text { padrão }\end{array}$} & \\
\hline & Média & $\begin{array}{c}\text { Desvio - } \\
\text { padrão }\end{array}$ & & & \\
\hline \multicolumn{6}{|c|}{ Proporção da duração (seg.) } \\
\hline Escuta & 0,67 & $(0,26)^{*}$ & 0,58 & $(0,25)^{* *}$ & 0,098 \\
\hline Elocução & 0,56 & $(0,25)$ & 0,39 & $(0,20)$ & $\mathbf{0 , 0 0 3}$ \\
\hline Total & 0,62 & $(0,25)$ & 0,50 & $(0,23)$ & 0,043 \\
\hline \multicolumn{6}{|c|}{ Freqüência/min. } \\
\hline Escuta & 12,69 & $(3,93)^{*}$ & 13,75 & $(4,14)$ & 0,366 \\
\hline Elocução & 24,47 & $(20,09)$ & 15,44 & $(6,69)$ & $\mathbf{0 , 0 1 7}$ \\
\hline Total & 14,16 & $(4,77)$ & 13,88 & $(4,40)$ & 0,804 \\
\hline \multicolumn{6}{|l|}{ Desvios/min } \\
\hline Escuta & 7,46 & $(4,55)$ & 10,69 & $(3,14)^{*}$ & 0,011 \\
\hline Elocução & 8,60 & $(6,53)$ & 8,82 & $(3,34)$ & 0,989 \\
\hline Total & 9,54 & $(4,97)$ & 10,78 & $(2,30)$ & 0,251 \\
\hline \multicolumn{6}{|c|}{ Duração média (seg.) } \\
\hline Escuta & 3,26 & $(1,48)^{* *}$ & 2,42 & $(0,91)^{* *}$ & $\mathbf{0 , 0 0 7}$ \\
\hline Elocução & 1,80 & $(1,10)$ & 1,55 & $(0,72)$ & 0,275 \\
\hline Total & 2,68 & $(1,13)$ & 2,07 & $(0,72)$ & 0,011 \\
\hline \multicolumn{6}{|l|}{ Piscar/min. } \\
\hline Escuta & 14,63 & $(10,17)^{*}$ & 15,24 & $(11,84)$ & 0,885 \\
\hline Elocução & 22,04 & $(12,95)$ & 18,40 & $(28,39)$ & 0,549 \\
\hline Total & 33,44 & $(18,21)$ & 32,42 & $(18,90)$ & 0,693 \\
\hline
\end{tabular}

$* p$ (fases) $<0,05 \quad * * p$ (fases) $<0,001$ 
Proporção da duração do olhar. A Tabela 1 mostra que os pacientes olharam por uma porcentagem de tempo maior para o interlocutor do que o grupo não-clínico, quanto às medidas globais $(F=4,28 ; p=0,04)$. Examinando cada fase, observa-se que esta diferença se concentrou na fase de elocução, na qual o grupo clínico apresentou maior proporção da duração do olhar do que o grupo não-clínico $(F=9,43 ; p=$ 0,003). Não houve diferenças significativas entre os grupos quanto a esta variável na fase de escuta $(F=2,82 ; p=0,098)$.

Freqüência do comportamento de olhar. Os resultados da Tabela 1 mostram que o grupo clínico apresentou também maior freqüência de olhar do que o grupo não-clínico na fase de elocução ( $F=5,99 ; p=0,02)$. Não houve diferenças significativas na fase de escuta $(F=0,82 ; p=0,36)$ nem nas medidas totais $(F=0,06 ; p=0,80)$. Os resultados sobre a duração e a freqüência do olhar, tomados conjuntamente, demonstram que os pacientes olhavam mais para o interlocutor do que o grupo não-clínico (GNC) enquanto falavam.

Fixidez do olhar: desvios de olhar e duração média do olhar. A Tabela 1 mostra que os pacientes desviaram menos o olhar do rosto do interlocutor ao escutá-lo do que os sujeitos do GNC ( $F=6,79 ; p=0,01)$. Não houve diferença significativa entre os grupos na fase de elocução $(F=0,00 ; p=0,98)$ ou no total das interações $(F=1,35 ; p=0,25)$. Os pacientes apresentaram também maior duração média dos olhares, ou seja, olhares mais prolongados, no total das quatro situações, do que o grupo não-clínico $(F=6,90 ; p=0,01)$. Examinando cada fase, observa-se que os olhares mais prolongados dos pacientes ocorreram na fase de escuta $(F=7,81 ; p=0,007)$. Não houve diferenças significativas na fase de elocução $(F=$ $1,21 ; p=0,27)$. Pode-se concluir, portanto, que os pacientes apresentaram um olhar mais fixo ao escutar, pois eles tinham olhares mais prolongados e desviavam menos o olhar do interlocutor do que os sujeitos do grupo não-clínico.

Comportamento de piscar. Os resultados da Tabela 1 mostram que não houve diferenças significativas entre os grupos clínico e não-clínico com relação a esta variável, na fase de escuta $(F=0,02 ; p=0,88)$ ou de elocução $(F=0,364$; $p=0,55)$, nem nas medidas globais $(F=0,66 ; p=3,51)$. Esta variável foi menos sensível para diferenciar os dois grupos.

Em resumo, observou-se diferenças significativas entre os grupos clínico e não-clínico em dois aspectos. Primeiro, os pacientes olharam mais em direção ao interlocutor, tanto em termos da freqüência quanto da duração do olhar, na fase de elocução. Em segundo lugar, foi observada uma maior fixidez do olhar dos pacientes, na fase de escuta.

Correlação entre duração e freqüência do olhar. Foi feita uma análise correlacional de Pearson entre a freqüência e a duração do olhar, para cada grupo de sujeitos. Ambos os grupos tiveram uma correlação significativa entre estas duas variáveis, ou seja, as pessoas que olhavam mais vezes olhavam também por mais tempo para o interlocutor, mas os pacientes apresentaram correlação muito menos elevada $(r=0,49 ; p=$ $0,003)$ dos que os sujeitos do grupo não clínico $(r=0,80 ; p=$ 0,00 ) entre estas as variáveis.

\section{Comparação entre as fases de escuta e de elocução}

Apresentaremos aqui as análises dos dados referentes às comparações entre as fases de escuta e de elocução das interações sociais, dispostos na Tabela 1.

Proporção da duração do olhar. Os resultados da Tabela 1 mostram que ambos os grupos, clínico $(t=-2,59 ; p=$ $0,01)$ e não-clínico ( $t=-7,29 ; p=0,00)$ olharam uma porcentagem de tempo maior para o interlocutor ao escutar do que ao falar, conforme teoricamente esperado. Entretanto, a diferença entre as duas fases foi menor para os pacientes, pois eles olharam para o interlocutor em 67\% do tempo da fase de escuta e 56\% do tempo da fase de elocução, uma diferença percentual de $11 \%$. Os sujeitos do grupo não-clínico olharam para o interlocutor em 58\% do tempo da fase de escuta e 39\% do tempo da elocução, uma diferença de 19\%. Além disso, os valores de $p$ mostram que a diferença entre fases foi mais significativa para o grupo não-clínico $(p=0,00)$ do que para o grupo clínico $(p=0,01)$. Estes resultados indicam que o comportamento de olhar dos pacientes foi menos diferenciado entre estas fases de escuta e de elocução das interações sociais.

Duração média de cada ocorrência de olhar. Os resultados da Tabela 1 mostram que ambos os grupos, clínico $(t=-5,89 ; p=0,00)$ e não-clínico $(t=-6,57 ; p=0,00)$, apresentaram olhares mais prolongados em direção ao interlocutor enquanto o escutavam do que enquanto falavam, tal como esperado.

Freqüência de desvios de olhar. A Tabela 1 mostra que o grupo clínico teve apenas uma tendência não significativa $(t=1,23 ; p=0,23)$ de desviar menos o olhar na escuta, enquanto o grupo não-clínico $(t=-2,31 ; p=0,03)$ apresentou uma freqüência significativamente maior de desvios de olhar na fase de escuta do que de elocução.

Freqüência do comportamento de piscar. A Tabela 1 mostra que os pacientes apresentaram uma freqüência muito menor de piscar na fase de escuta do que de elocução ( $t=$ $3,51 ; p=0,002)$, o que não ocorreu com o grupo não-clínico ( $t$ $=0,55 ; p=0,59)$.

Freqüencia do comportamento de olhar. Os resultados da Tabela 1 mostram que apenas o grupo clínico $(t=3,43$; $p=$ 0,002 ) apresentou um número significativamente menor (quase a metade) de olhar por minuto na fase de escuta do que de elocução, o que não ocorreu com o grupo não-clínico $(t=$ $1,72 ; p=0,09)$.

Os resultados descritos acima indicam que os pacientes apresentaram uma maior imobilidade ou fixidez do olhar na 
fase de escuta do que de elocução, pois além de olhar mais tempo e com olhares mais prolongados para o interlocutor, eles piscaram muito menos vezes por minuto na fase de escuta do que na fase de elocução, além de apresentar uma freqüência menor de olhares por minuto, sem ter desviado mais o olhar.

\section{Comparação entre os tipos de situação: fazer e} receber crítica

Os dados comparativos das situações de fazer e receber crítica estão apresentados na Tabela 2.

Freqüência de desvios de olhar. A Tabela 2 mostra que ambos os grupos tiveram maior número de desvios de olhar do interlocutor nas situações de receber crítica do que de fazer crítica. Cada grupo apresentou este resultado em uma fase diferente. Os pacientes apresentaram esta diferença na fase de elocução ( $t=2,24 ; p=0,03)$ e o grupo não-clínico, na fase de escuta $(t=2,50 ; p=0,01)$.

Freqüência do comportamento de olhar. Os resultados da Tabela 2 mostram que as situações de receber crítica provocaram uma menor freqüência do comportamento de olhar o interlocutor do que as situações de fazer crítica. Entretanto, esta diferença só foi observada no caso dos pacientes ( $t=$ 2,03; $p=0,05$ ), na fase de escuta da interação social.

Duração média de cada ocorrência de olhar. Os dados da Tabela 2 mostram que apenas os pacientes apresentaram olhares mais prolongados ao escutar o interlocutor nas situações de receber crítica do que nas situações de fazer crítica $(t=2,12 ; p=0,04)$.

Em resumo, os resultados mostram que ambos os grupos apresentaram menos olhar nas situações de receber crítica. Os pacientes reagiram mais a estas situações, porque eles apresentaram mudanças em três parâmetros diferentes do comportamento, enquanto o grupo não-clínico o fez apenas em um. Além disso, os pacientes apresentaram diferenças significativas em ambas as fases da situação de receber crítica, enquanto que o grupo não-clínico apresentou diferença significativa apenas em uma fase da interação.

Demais parâmetros. A proporção da duração do olhar não mostrou diferença significativa entre as situações de receber ou fazer crítica. Para o comportamento de piscar, não foi possível fazer este tipo de comparação, pois este comportamento só foi observado nas situações 1 e 3, ambas de receber crítica.

Tabela 2

Médias e desvios-padrão dos parâmetros do comportamento de olhar dos grupos clínico e não-clínico, nas fases de escuta e de elocução, em função do tipo de situação

Parâmetros do comportamento de olhar

\begin{tabular}{|c|c|c|c|c|c|c|c|c|}
\hline & \multicolumn{4}{|c|}{ Grupo clínico } & \multicolumn{4}{|c|}{ Grupo não clínico } \\
\hline & \multicolumn{2}{|c|}{ Receber críticas } & \multicolumn{2}{|c|}{ Fazer críticas } & \multicolumn{2}{|c|}{ Receber críticas } & \multicolumn{2}{|c|}{ Fazer críticas } \\
\hline & Média & $\begin{array}{l}\text { Desvio } \\
\text { padrão }\end{array}$ & Média & $\begin{array}{l}\text { Desvio } \\
\text { padrão }\end{array}$ & Média & $\begin{array}{l}\text { Desvio } \\
\text { padrão }\end{array}$ & Média & $\begin{array}{l}\text { Desvio } \\
\text { padrão }\end{array}$ \\
\hline \multicolumn{9}{|c|}{ Proporção duração (seg.) } \\
\hline Escuta & 0,67 & $(0,27)$ & 0,58 & $(0,29)$ & 0,57 & $(0,27)$ & 0,38 & $(0,23)$ \\
\hline Elocução & 0,66 & $(0,28)$ & 0,54 & $(0,27)$ & 0,55 & $(0,31)$ & 0,40 & $(0,23)$ \\
\hline \multicolumn{9}{|c|}{ Freqüência/min. } \\
\hline Escuta & 12,10 & $(3,97)$ & 13,83 & $(5,50)^{*}$ & 13,50 & $(4,13)$ & 13,81 & $(5,49)$ \\
\hline Elocução & 31,84 & $(35,21)$ & 22,63 & $(21,00)$ & 14,22 & $(6,17)$ & 16,10 & $(8,67)$ \\
\hline \multicolumn{9}{|c|}{ Desvios/min } \\
\hline Escuta & 7,87 & $(5,06)$ & 7,44 & $(5,81)$ & 11,06 & $(3,84)$ & 9,02 & $(4,20)^{*}$ \\
\hline Elocução & 11,31 & $(12,34)$ & 6,82 & $(5,62)^{*}$ & 8,17 & $(4,81)$ & 8,98 & $(3,53)$ \\
\hline \multicolumn{9}{|c|}{ Duração média (seg.) } \\
\hline Escuta & 3,55 & $(1,87)$ & 2,98 & $(1,49)^{*}$ & 2,51 & $(1,20)$ & 2,35 & $(1,20)$ \\
\hline Elocução & 1,74 & $(1,25)$ & 2,01 & $(1,21)$ & 1,56 & $(0,79)$ & 1,52 & $(0,91)$ \\
\hline
\end{tabular}

$* p<0,05$ 


\section{Comparação entre tipos de interlocutores: do sexo masculino e feminino}

Os resultados a respeito do gênero do interlocutor estão na Tabela 3. Observa-se que houve menos comportamento de olhar nas situações com interlocutor masculino.

Proporção da duração do olhar. A Tabela 3 mostra que, para ambos os grupos, houve uma menor proporção da duração do olhar nas situações com interlocutor masculino do que feminino. No caso dos pacientes, esta diferença se manifestou apenas na fase de escuta $(t=2,19 ; p=0,036) \mathrm{e}$ para o grupo não-clínico ocorreu uma diferença significativa na fase de escuta $(t=3,40 ; p=0,002)$ e na fase de elocução $(t$ $=2,64 ; p=0,013)$.

Freqüência do comportamento de olhar. A Tabela 3 mostra que os pacientes apresentaram um número significativamente menor de olhares por minuto $(t=2,22$; $p=$ $0,034)$ nas situações com interlocutor masculino do que feminino, na fase de elocução. Na fase de escuta, não houve diferença significativa em função do gênero. O grupo não- clínico não apresentou diferenças significativas em função do gênero, para esta variável.

Freqüência de desvios de olhar. Pode-se observar, na Tabela 3, que o grupo não-clínico apresentou uma maior freqüência de desvios de olhar nas situações com interlocutor masculino do que feminino ( $t=-2,81 ; p=0,008)$, apenas na fase de escuta. O grupo clínico não apresentou esta diferença em função do gênero.

Freqüencia do comportamento de piscar. A Tabela 3 mostra que os pacientes apresentaram um número significativamente maior de piscadas por minuto diante do interlocutor masculino do que feminino, na fase de escuta $(t=$ $-3,06 ; p=0,005)$ e na medida global $(t=3,19 ; p=0,004)$. Para o grupo não-clínico, não houve diferenças significativas em função do gênero do interlocutor.

Duração média de cada ocorrência de olhar. A Tabela 3 mostra que ocorreram olhares menos prolongados em direção ao interlocutor masculino do que feminino, na fase de

Tabela 3

Médias e desvios-padrão dos parâmetros do comportamento de olhar dos grupos clínico e nãoclínico, nas fases de escuta e de elocução, em função do gênero do interlocutor

\begin{tabular}{lcccccccc}
\hline & \multicolumn{7}{c}{ Parâmetros do comportamento de olhar } \\
\cline { 2 - 8 } & \multicolumn{7}{c}{ Interlocutor do grupo clínico } & \multicolumn{2}{c}{ Interlocutor do grupo não clínico } \\
\cline { 2 - 8 } & \multicolumn{2}{c}{ Feminino } & \multicolumn{2}{c}{ Masculino } & \multicolumn{2}{c}{ Feminino } & Masculino \\
\cline { 2 - 8 } & Média & $\begin{array}{c}\text { Desvio } \\
\text { padrão }\end{array}$ & Média & $\begin{array}{c}\text { Desvio } \\
\text { padrão }\end{array}$ & Média & $\begin{array}{c}\text { Desvio } \\
\text { padrão }\end{array}$ & Média & $\begin{array}{c}\text { Desvio } \\
\text { padrão }\end{array}$ \\
\hline Proporção duração (seg.) & & & & & & & & \\
$\quad$ Escuta & 0,70 & $(0,27)$ & 0,62 & $(0,29)^{*}$ & 0,60 & $(0,28)$ & 0,50 & $(0,28)^{*}$ \\
$\quad$ Elocução & 0,59 & $(0,27)$ & 0,53 & $(0,29)$ & 0,44 & $(0,22)$ & 0,35 & $(0,22)^{*}$ \\
Freqüência/min. & & & & & & & & \\
Escuta & 12,50 & $(4,64)$ & 12,96 & $(4,45)$ & 13,04 & $(4,22)$ & 14,29 & $(5,49)$ \\
Elocução & 28,90 & $(28,51)$ & 22,22 & $(17,30)^{*}$ & 17,72 & $(6,23)$ & 14,32 & $(12,06)$ \\
Desvios/min & & & & & & & & \\
$\quad$ Escuta & 7,01 & $(5,13)$ & 8,51 & $(5,21)$ & 9,21 & $(4,01)$ & 11,72 & $(4,51)^{*}$ \\
Elocução & 8,09 & $(8,11)$ & 9,55 & $(8,13)$ & 9,19 & $(3,90)$ & 7,92 & $(4,31)$ \\
Piscar/min & & & & & & & & \\
Escuta & 12,63 & $(10,20)$ & 17,62 & $(11,87)^{*}$ & 14,45 & $(11,45)$ & 16,44 & $(14,64)$ \\
$\quad$ Elocução & 23,05 & $(29,30)$ & 21,94 & $(13,37)$ & 21,03 & $(24,96)$ & 17,48 & $(32,83)$ \\
Duração média (seg.) & & & & & & & & \\
$\quad$ Escuta & 3,65 & $(1,68)$ & 2,98 & $(1,65)^{*}$ & 2,73 & $(1,20)$ & 2,05 & $(1,08)^{*}$ \\
Elocução & 1,85 & $(1,35)$ & 1,97 & $(1,32)$ & 1,48 & $(0,10)$ & 1,66 & $(1,26)$ \\
\hline
\end{tabular}

$* p<0,05$ 
escuta, tanto para os pacientes $(t=3,55 ; p=0,001)$, quanto para o grupo não clínico $(t=2,69 ; p=0,01)$.

Em resumo, os resultados referentes ao gênero do interlocutor mostraram que ambos os grupos olharam menos para o interlocutor do sexo masculino do que feminino, em termos de diversos parâmetros. Os pacientes variaram o comportamento de olhar em termos de quatro parâmetros e o grupo não-clínico reagiu em termos de três parâmetros.

\section{Discussão e conclusões}

Os resultados obtidos aqui mostraram, em primeiro lugar, que o comportamento de olhar dos pacientes diferiu significativamente do grupo de pessoas sem história psiquiátrica, em termos de dois parâmetros. O primeiro se refere à proporção da duração e à freqüência do olhar. Os pacientes olharam mais tempo e mais freqüentemente enquanto falavam com o interlocutor do que o grupo não-clínico. Eles apresentaram ainda olhares mais prolongados ao escutar o interlocutor do que o grupo não clínico.

Esta diferença encontrada entre os dois grupos confirma os dados obtidos por Rutter (1976), que também encontrou maior duração do olhar no grupo de pacientes psicóticos, utilizando situações sociais cotidianas. Por outro lado, estes resultados contradizem os dados de estudos de caso com entrevistas psiquiátricas e sugerem que a baixa freqüência e duração do olhar observados nas entrevistas psiquiátricas resultam de fatores específicos a estas situações, tais como o status do interlocutor e o conteúdo pessoal altamente significativo e emocional tratado, que são embaraçosos e geram ansiedade (Argyle \& Dean,1965; Argyle \& Kendon,1967). Situações deste tipo seriam, segundo Beaulieu (1996), pouco representativas de situações sociais cotidianas e, portanto, inadequadas metodologicamente para serem utilizadas em pesquisas sobre habilidades sociais, pois não possibilitam generalização dos resultados.

O segundo parâmetro da diferença significativa observada entre os grupos, no presente trabalho, se refere à fixidez do olhar dos pacientes, comparativamente ao grupo não-clínico. Esta característica ocorreu na fase de escuta, na qual os pacientes desviaram menos o olhar do interlocutor ao mesmo tempo em que apresentaram olhares mais prolongados. Estes resultados confirmam os dados de Beaulieu (1996), que observou também menos desvios de olhar em pacientes psiquiátricos, em situações sociais semelhantes às utilizadas aqui, concluindo que eles apresentavam um olhar mais fixo.

No que se refere às relações entre os componentes estudados, os pacientes apresentaram também uma divergência em relação ao grupo não-clínico, pois obtiveram uma correlação menos estreita entre a freqüência e a duração do olhar. Segundo Argyle (1967/1994), as pessoas que olham mais vezes também olham mais tempo, o que acarreta uma correlação elevada entre estas duas variáveis, tipicamente encontrada na população geral. Uma correlação elevada só foi observada aqui para o grupo não-clínico, cujo coeficiente de correlação foi quase o dobro do observado para os pacientes.
Quanto às relações entre as fases de escuta e de elocução das interações sociais, os resultados mostraram a inversão esperada entre os comportamentos de olhar e de falar, para ambos os grupos de sujeitos. Ambos olharam mais tempo para o interlocutor e com olhares mais prolongados na fase de escuta do que de elocução, o que é tipicamente encontrado nas pesquisas desta área (Argyle \& Kendon, 1967; Caballo, 1996; Trower, 1980). O que foi particular no desempenho dos pacientes é que eles apresentaram uma diferença menor (11\%) entre as fases do que o grupo não-clínico (19\%). Este resultado confirma dados obtidos por Trower (1980) e refletem a dificuldade dos pacientes de monitorar estímulos sociais dos comportamentos do interlocutor e de monitorar seus próprios comportamentos, o que resulta em menor diferenciação entre as fases (Trower, 1980).

Um outro aspecto divergente observado no comportamento de olhar dos pacientes é que eles olharam para o interlocutor durante uma proporção de tempo que ultrapassa a faixa usualmente esperada para a fase de elocução. Nesta fase, as pessoas usualmente olham para o interlocutor 30\% a 50\% do tempo (Argyle \& Kendon, 1967; Caballo, 1996). No presente trabalho, os resultados globais mostraram que os pacientes olharam para o interlocutor $56 \%$ da fase de elocução, acima da faixa esperada. O grupo nãoclínico, ao contrário, olhou para o interlocutor durante 39\% do tempo de elocução, dentro da faixa esperada. Este resultado indica que o comportamento de olhar dos pacientes não se mostrou compatível com a fase de elocução, onde se requer a elaboração cognitiva necessária para construir respostas de solução de problemas interpessoais. Nesta fase, olhar menos para o interlocutor teria a função adaptativa de reduzir a quantidade de informação externa concomitante, favorecendo a elaboração cognitiva (Argyle \& Dean,1965; Kendon,1967).

As características dos pacientes descritas acima foram identificadas em relação a um grupo de referência na comunidade, estando controlados os fatores referentes ao nível sócio-econômico e educacional e faixa etária, o que confere maior validade social a estes resultados, pois foram calibrados pelas características da população de referência dos pacientes na comunidade (Kazdin, 1977; Torgrud \& Holborn, 1992).

No que se refere ao comportamento de olhar frente a mudanças nas demandas das situações, os resultados mostraram que as situações de receber crítica resultaram em uma maior freqüência de desvios do olhar do que as de fazer críticas, para ambos os grupos, assim como uma menor freqüência do comportamento de olhar para os pacientes, embora eles tenham apresentado, por outro lado, olhares mais prolongados. Estes resultados sugerem, em geral, uma maior dificuldade dos sujeitos nas situações de receber críticas. Esta interpretação está em acordo com dados de outros autores, segundo os quais as situações que envolvem processos emocionais ou questões cognitivamente mais difíceis provocam mais desvios de olhar do interlocutor (Argyle \& Kendon, 1967), assim como as situações envolvendo maior tensão entre o sujeito e o interlocutor (Argyle \& Dean, 1965). Segundo Argyle (1984), os pacientes 
psiquiátricos, em particular o esquizofrênico, se mostram particularmente sensíveis a situações em que são criticados.

Os resultados do comportamento de olhar em situações de receber crítica são compatíveis também com dados de pesquisas realizadas em nosso meio. Por exemplo, já se observou uma maior latência das respostas, entonação de voz menos firme, maior freqüência e duração de silêncios e menor duração da fala dos sujeitos nas situações de receber crítica do que de fazer críticas (Bandeira et al., 2002; Tremblay \& Bandeira, 2001), assim como uma menor freqüência de respostas verbais assertivas (Bandeira et al., 2002) e uma cota global inferior de assertividade verbal e não-verbal (Bandeira \& Ireno, no prelo).

Os resultados obtidos aqui mostraram também que o comportamento de olhar variou significativamente em função do gênero do interlocutor. A proporção da duração do olhar, assim como a duração média de cada olhar de ambos os grupos foi menor frente ao interlocutor do sexo masculino do que feminino, na fase de escuta. No caso do grupo nãoclínico, observou-se ainda uma menor proporção da duração do olhar ao falar com o interlocutor masculino e mais desvios de olhar por minuto ao escutar o interlocutor masculino do que feminino. No caso dos pacientes, houve também uma maior freqüência de piscar quando eles escutavam o interlocutor masculino e uma menor freqüência de olhar quando eles falavam com o interlocutor do sexo masculino do que feminino.

Os resultados referentes ao gênero do interlocutor são coerentes com dados obtidos para outros aspectos do comportamento. Já foi observado, por exemplo, uma maior proporção da duração da fala e uma menor freqüência de silêncios diante do interlocutor masculino do que feminino (Bandeira et al., 2002). Estes resultados são coerentes porque a proporção do tempo da fala e do tempo do olhar tendem a ser inversamente relacionados (Argyle \& Dean, 1965; Argyle \& Kendon, 1967). Quanto ao conteúdo da fala, já foi observada uma maior freqüência de respostas verbais assertivas de expressar necessidade e insatisfação e de dar uma solução para o problema discutido com o interlocutor masculino do que feminino (Bandeira et al., 2002). Estes dados mostram uma relação inversa entre o comportamento de olhar e a freqüência de respostas assertivas e de solução de problemas, pois os sujeitos que falam mais tempo são os mais assertivos. O comportamento de olhar menos para o interlocutor masculino pode refletir, portanto, maior elaboração cognitiva para construção e emissão de uma resposta verbal mais elaborada e mais assertiva.

Os resultados obtidos no presente trabalho confirmaram algumas relações encontradas em amostras de outras culturas indicando, portanto, que as características dos pacientes psiquiátricos discutidas acima independem de diferenças culturais. Além disso, os resultados tem implicações práticas para intervenções clínicas envolvendo estes pacientes, em particular os programas de readaptação psicossocial que visam a sua reinserção social na comunidade. Os resultados sugerem uma orientação para o treinamento das habilidades sociais, no que se refere ao comportamento de olhar em uma interação social.

\section{Agradecimentos}

Esta pesquisa foi financiada pela FAPEMIG (Fundação de Amparo a Pesquisa do Estado de Minas Gerais) e pelo CNPq (Conselho Nacional de Desenvolvimento Científico e Tecnológico).

\section{Referências}

Argyle, M. (1984). Social skills and the analysis of situations and conversations. In C. R. Hollin \& P. Trower (Orgs.), Handbook of social skills training: clinical applications and new directions (pp.185-216). Nova York: Pergamon Press.

Argyle, M. (1994). Psicologia del comportamento interpessoal. Madrid: Alianza Universidad. (Texto original publicado em 1967)

Argyle, M., \& Dean, J. (1965). Eye contact, distance and affiliation. Sociometry, 28, 289-304.

Argyle, M., \& Kendon, A. (1967). The experimental analyses of social performance. Advances in Experimental Social Psychology, 3, 55-98.

Bandeira, M. (1991). Desinstitucionalização ou transinstitucionalização: lições de alguns países. Psicologia: Teoria e Pesquisa, 6 (2), 171-182.

Bandeira, M., Cardoso, C. S., Fernandes, M. L., Resende, R. A., \& Santos, S. C. A. S. (1998). Competência social de psicóticos: validação social de habilidades específicas. Jornal Brasileiro de Psiquiatria, 47(5), 217-226.

Bandeira, M., \& Dorvil, H. (1996). Les Consultations a la salle d' urgence psiquiatrique: comparaison entre des echantillons brésiliens et québecois. Le Médecin du Québec, 31(8), 59-69.

Bandeira, M., \& Ireno, E. (no prelo). Reinserção social de psicóticos: avaliação global do grau de assertividade em situações de fazer e receber críticas. Psicologia: Reflexão e Crítica.

Bandeira, M., Machado, E. L., \& Pereira, E. A. A. (2002). Reinserção social de psicóticos: componentes verbais e não-verbais do comportamento assertivo, em situações de fazer e receber críticas. Psicologia: Reflexão e Crítica, 15(1), 89-104.

Bandeira, M., \& Tremblay, L. (1998). Competência social de psicóticos: um estudo de validação social. Jornal Brasileiro de Psiquiatria, 47(4), 185192.

Beaulieu, S. (1996). La composante regard dans l'entraînement aux habiletés sociales auprès des personnes souffrant de schizophrenie. Dissertação de mestrado não-publicada, Université de Montréal, Canadá.

Bellack, A. S. (1983). Recurrent problems in the behavioral assessment of social skill. Behavior Research and Therapy, 21(1), 29-41.

Bellack, A. S., Morrison, R. L., Mueser, K. T., \& Wade, J. H. (1989). Social competence in schizoaffective disorder, bipolar disorder, and negative and non-negative schizophrenia. Schizophrenia Research, 2, 391-401.

Bellack, A. S., Morrison, R. L., Mueser, K. T, Wade, J. H., \& Sayer, S. L. (1990). Role play for assessing the social competence of psychiatric patients. Journal of Consulting and Clinical Psychology, 2(3), 248-255.

Caballo, V. E. (1996). Manual de técnicas de terapia e modificação do comportamento. São Paulo: Santos Livraria.

Cyr, M., Hodgins, S., Gaston, L. E., \& Viens, L. (1990). La vie au sein d'appartements surveillés pour patients psychiatriques chroniques. Revue Canadienne de Santé Mentale Communautaire, 9(1), 23-37.

Del Prette, Z. A. P., \& Del Prette, A. (1999). Psicologia das habilidades sociais: terapia e educação. Rio de Janeiro: Vozes.

Donahoe, C. P., \& Drisienga, S. A.(1988). A review of social skills training with chronic mental patients. In M. Hersen, R.M. Eisler, \& P. M. Miller (Orgs.), Progress in behavioral modification (pp.131-164). Newbury Park: 
Sage.

Dorvil, H. (1987). Les caracteristiques du syndrome de la porte tournante à l'hôpital Louis H. Lafontaine. Santé Mentale au Québec, XII(1), 70-89.

Eisler, R. M., Hersen, M., Miller, P. M., \& Blanchard, E. B. (1975). Situational determinants of assertive behaviors. Journal of Consulting and Clinical Psychology, 43(3), 330-340.

Foy, D. W., Wallace, C. J., \& Liberman, R. P. (1983). Advances in social skills training for chronic mental patients. Advances in Clinical Behavioral Analysis, 11, 232-248.

Gambrill, E. D. (1978). Behavior modification: handbook of assessment, intervention and evaluation. Washington: Jossey-Bass Publishers.

Hogarty, G. E., Anderson, C. M., Reiss, D. J., Kornblith, S. J., Greenwald, D. P., Ulrich, R. F., \& Carter, M. (1986). Family psychoeducation, social skills training, and maintenance chemotherapy in the aftercare treatment of schizophrenia. Archives of General Psychiatry, 48, 340-347.

Kazdin, A. E. (1977). Assessing the clinical or applied importance of behavior change through social validation. Behavior Modification, 1, 427-451.

Kendon, A. (1967). Some functions of gaze direction in social interaction. Acta Psychologica, 26, 22-63.

Larsen, R. J., \& Shackelford, T. K. (1996). Gaze avoidance: personality and social judgments of people who avoid direct face-to-face contact. Personality and Individual Differences, 21(6), 907-917.

Liberman, R. P. (1984). Social skills training with relapsing schizophrenics: an experimental analysis. Behavior Modification, 8(2), 155-173.

Liberman, R. P., Wallace, C. J., Blackwell, C. A., Eckman, T. A., Vaccaro, J. V., \& Kuehnel, T. G. (1985). Innovations in skills training for the seriously mentally ill: the UCLA social and independent living skills modules. Innovations and Research, 2, 43-60.

Merluzzi, T. V., \& Biever, J. (1987). Role-playing procedures for the behavioral assessment of social skill: a validity study. Behavioral Assessment, 9, 361-377.

Moos, R. H. (1968). Situational analysis of a therapeutic community milieu. Journal of Abnormal Psychology, 73, 49-61.

Mueser, K. T., Bellack, A. S., Douglas, M. S., \& Morrison, R. L. (1991). Prevalence and stability of social skills deficits in schizophrenia. Schizophrenia Research, 5, 167-176.

Mueser, K. T., Bellack, A S., Morrison, R. L., \& Wixted, J. T. (1990a). Social competence in schizophrenia: premorbid adjustment, social skill and domains of functioning. Journal of Psychiatric.Research, 24(1), 5163.
Mueser, K. T, Bellack, A. S., Morrison, R. L., \& Wade, J. H. (1990b). Gender, social competence and symptomatology in schizophrenia: a longitudinal analysis. Journal of Abnormal Psychology, 99(2), 138-147.

Mueser, K. T., Deonan, R., Penn, D. L. Blanchard, J. J., Bellack, A. S., Nishith, P., \& DeLeon, J. (1996). Emotion recognition and social competence in chronic schizophrenia. Journal of Abnormal Psychology, 105(2), 271-275.

Mueser, K. T., Wallace, C. J., \& Liberman, R. P. (1995). New developments in social skills training. Behavior Change, 12(1), 31-40.

Penny, N. H., Mueser, K. T., \& North, C. T. (1995). The Allen Cognitive Level test and social competence in adult psychiatric patients. American Journal of Occupational Therapy, 49(5), 420-427.

Rutter, D. R. (1976). Visual interaction in recently admitted and chronic longstay schizophrenic patients. British Journal of Social and Clinical Psychology, 15, 295-303.

Shepperd, G. (1986). Social skills training and schizophrenia. In C. R. Hollin \& P. Trower (Orgs.), Handbook of social skills training: clinical applications and new directions (pp. 9-37). Nova York: Pergamon Press.

Torgrud, L. J., \& Holborn, S. W. (1992). Developing externally valid roleplay for assessment of social skills: a behavior analytic perspective. Behavioral Assessement, 1414, 245-277.

Tremblay, L. (1992). Evaluation de la competence sociale de personnes souffrant de troubles mentaux graves: études de validation sociales. Relatório final de pesquisa apresentado ao Conseil Québecois de la Recherche Sociale (RS-1725-089), Canadá.

Tremblay, L., \& Bandeira, M. (1989). L'évaluation ecologique des habilétes sociales (EEHS). Documento não-publicado, Institut Pinel de Montréal, Canadá.

Tremblay, L., \& Bandeira, M. (2001). L’Influence du type de situation sur la competence social: étude de comparaison sociale. Cadernos de Psicologia, 10, 71-88.

Trower, P. (1980). Situational analysis of the components and processes of behavior of socially skilled and unskilled patients. Journal of Consulting and Clinical Psychology, 48, 327-339.

Marina Bandeira, Doutora pela Université de Montréal (Canadá), é Professora Adjunta na Universidade Federal de São João Del Rei (UFSJ) e Pesquisadora do CNPq.

Elaine Leandro Machado é psicóloga pelo Curso de Psicologia da UFSJ.

Sabrina Martins Barroso, Tais Ribeiro Gaspar e Magali Milene Silva são alunas do Curso de Psicologia da UFSJ, bolsistas de iniciação científica do CNPq.

Endereço para correspondência: Departamento de Psicologia - UFSJ; Praça Dom Helvécio, 74; São João del Rei, MG; CEP 36.300-000.E-mail: bandeira@funrei.br 